\title{
Anabases
}

ANABASES Traditions et réceptions de l'Antiquité

$21 \mid 2015$

Varia

\section{La poésie didactique dans l'Antiquité : une invention des Modernes}

\section{Pierre Vesperini}

\section{(2) OpenEdition}

1 Journals

Édition électronique

URL : http://journals.openedition.org/anabases/5207

DOI : 10.4000/anabases.5207

ISSN : 2256-9421

Éditeur

E.R.A.S.M.E.

\section{Édition imprimée}

Date de publication : 1 avril 2015

Pagination : 25-38

ISSN : 1774-4296

\section{Référence électronique}

Pierre Vesperini, «La poésie didactique dans l'Antiquité : une invention des Modernes », Anabases [En ligne], 21 | 2015, mis en ligne le 01 avril 2018, consulté le 20 octobre 2019. URL : http:// journals.openedition.org/anabases/5207 ; DOI : 10.4000/anabases.5207 


\section{La poésie didactique dans l'Antiquité: une invention des Modernes}

Pierre VesperinI

L

a catégorie de «poésie didactique» est une catégorie couramment employée à propos de poèmes tels que Les Travaux et les Jours d'Hésiode, des poèmes présocratiques comme Sur la nature de Parménide ou d'Empédocle, des poèmes hellénistiques comme les Phénomènes d'Aratos ou les Thériaques de Nicandre, ou encore à propos de poèmes latins comme le De rerum natura de Lucrèce, les Géorgiques, ou les Astronomiques de Manilius.

Or, comme on l'a remarqué depuis longtemps, cette catégorie n'existait pas dans l'Antiquité'. Elle n'existait pas non plus à la Renaissance, ni dans la poésie de l'âge

1 J. Warton, Reflections on Didactic Poetry, Londres, I753, p. 29I: “The ancients have left us no rules or observations concerning this species of poetry". On lit parfois qu'Aristote, en disant que le poème d'Empédocle était un "discours versifié», aurait reconnu dans ce poème de

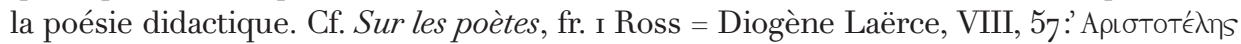

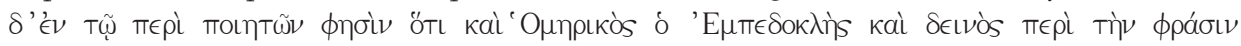
$\gamma^{\prime} \gamma_{\gamma} \nu \epsilon$, «Aristote, dans son écrit sur les poètes, dit qu'Empédocle est également homérique et s'est avéré un maître du verbe». Or, d'une part, cette façon de décrire le poème d'Empédocle est très tendancieuse, caractéristique de l'attitude d'Aristote, qui, comme il l'a fait pour les tragédies, essaie souvent de dégager des œuvres qu'il lit tout ce qui peut rappeler leurs conditions d'énonciation, pour en extraire un contenu: dans les tragédies, c'était l'intrigue, le muthos; dans le poème d'Empédocle, c'est le logos. Et, d'autre part, Aristote, loin d'appliquer la notion de “poésie didactique», exclut au contraire Empédocle de la poésie, pour le rattacher au discours philosophique. L'idée d'Aristote est d'ailleurs assez isolée dans l'Antiquité, mais on la retrouve chez Plutarque, de audiendis poetis, $16 c$ : Tà

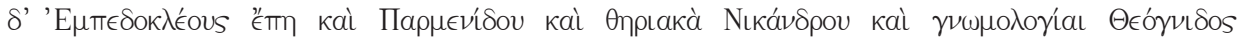

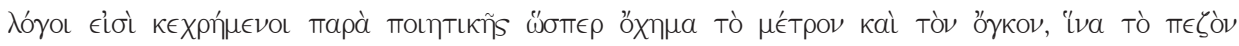


classique $^{2}$. La question que je souhaiterais poser dans cet article est donc double: d'où vient que nous parlons de "poésie didactique», et comment les Anciens parlaient-ils des poèmes que nous considérons, nous, comme «didactiques »?

Un lecteur moderne aura facilement l'impression qu'il y a entre Hésiode et Homère, ou entre le De rerum natura et l'Énéide, une différence de genre. Ce que je voudrais essayer de montrer, c'est que cette impression est déterminée par le «sol silencieux », pour le dire avec Foucault, à partir duquel sont produits nos discours. Elle repose en effet sur la distinction moderne entre « description » et « narration ». On aurait d'un côté des poèmes qui racontent des histoires, comme l'Iliade ou

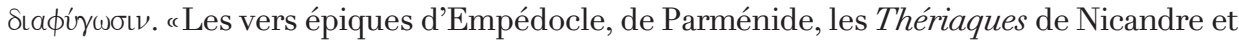
les discours gnomiques de Théognis sont des discours qui ont en quelque sorte emprunté le char de la poésie, c'est-à-dire son mètre et sa grandeur, afin d'éviter la prose.» On retrouve la même attitude à la Renaissance chez Castelvetro, éditeur et commentateur de la Poétique d'Aristote, estimant que, si Virgile n'avait écrit que les Géorgiques, il ne devrait pas être compté au nombre des poètes. On cite également pour justifier l'emploi de la catégorie de "poésie didactique» deux grammairiens tardifs, Diomède et Servius. Servius, dans son prologue du commentaire aux Géorgiques: Et hi libri didascalici sunt, unde necesse est, ut ad aliquem scribantur. Nam praeceptum et doctoris et discipulipersonam requirit: unde ad Maecenatem scribit sicut Hesiodus ad Persen, Lucretius ad Memmium. Mais Servius ne dit pas pour autant que la poésie didascalique est un genre à part, c'està-dire que le caractère didactique détermine une poétique différente de la poétique de l'Énéide: tout Virgile est "plein de science» (scientia plenus), comme il le dit au début de son commentaire du livreVI de l'Énéide, en faisant remonter la tradition de cette poésie sapientielle à Homère: Totus quidem Vergilius scientia plenus est, in qua hic liber possidet principatum, cuius ex Homero pars maior est. et dicuntur aliqua simpliciter, multa de historia, multa per altam scientiam philosophorum, theologorum, Aegyptiorum, adeo ut plerique de his singulis huius libri integras scripserint pragmatias. «Dans tout Virgile, on trouve de la science, et dans ce domaine ce livre tient le premier rang, dont la plus grande partie vient d'Homère. Pour le reste, certaines choses sont dites simplement, beaucoup sont tirées de l'Histoire, beaucoup sont tirées de la science sublime des philosophes, des théologiens, des Égyptiens, au point que de nombreux auteurs ont consacré des commentaires entiers sur chacun de ces différents éléments. » Chez Diomède, le qualificatif didascalice est également une étiquette, à l'intérieur d'une taxinomie complexe, véritable “système à tiroirs » pour le dire avec E.R. Curtius (La Littérature européenne et le Moyen Âge latin, tr. fr., Paris, I99I [I953²], p.688-692), qui montre bien comment le classement n'a rien à voir avec les classements qui nous sont familiers. Mais on ne peut en tirer que les Anciens distinguaient une poétique didactique d'une poétique «narrative », comme le fait K. Volk (The Poetics of Latin Didactic: Lucretius, Vergil, Ovid, Manilius, Oxford, 2002), qui fait un grave contresens sur l'expression "genus enarratiuum », lequel ne signifie pas "genre narratif », mais "genre explicatif, herméneutique».

2 Elle n'apparaît par exemple ni chez Du Bellay ni chez Ronsard ni chez Boileau. 
l'Énéide, et de l'autre des poèmes qui décrivent: Aratos décrit les constellations, Nicandre les différents types de bêtes venimeuses, etc. Cette première distinction recouvre une autre distinction familière aux modernes: celle entre fiction et science. Les poèmes qui racontent des histoires sont rangés du côté de la fiction, ceux qui décrivent la nature (ou des parties de la nature) sont rangés du côté de la science.

C'est ce partage moderne entre une poésie descriptive, scientifique, et une poésie narrative, fictionnelle, qui va fonder la distinction moderne entre poésie didactique et poésie épique. Par “poésie didactique», les savants modernes entendent en effet un genre poétique particulier, dans lequel la poésie sert de moyen à la communication d'un savoir. Les exposés qu'on rencontre sur les caractéristiques du genre didactique l'opposent souvent à la “poésie épique », qui n'aurait pas pour fin d'enseigner mais de divertir, en communiquant non pas un savoir, mais des histoires. On oppose ainsi couramment Homère et Hésiode, en disant que l'un est le fondateur de l'épopée et l'autre le fondateur de la poésie didactique. L'un raconterait des histoires, donc serait plutôt du côté de la fiction, l'autre communiquerait des enseignements, donc serait plutôt du côté de la sagesse, voire de la philosophie. L’opposition entre "poésie didactique et “poésie épique» détermine ainsi une série d'oppositions :

\begin{tabular}{|l|l|}
\hline poésie didactique & poésie épique \\
\hline description & narration \\
\hline $\begin{array}{l}\text { instruction (et plus généralement philosophie, } \\
\text { science) }\end{array}$ & poésie (et plus généralement art, littérature) \\
\hline savoir, vérité, science, philosophie, sagesse & histoires, mythes, fiction, littérature \\
\hline sérieux, sévérité & séduction, plaisir \\
\hline utilité & jeu, donc inutilité \\
\hline Hésiode & Homère \\
\hline $\begin{array}{l}\text { Aratos (Phénomènes), Nicandre (Thériaques, } \\
\text { Alexipharmakes) }\end{array}$ & Apollonius de Rhodes, Argonautiques \\
\hline Lucrèce, Géorgiques, Manilius... & $\begin{array}{l}\text { Virgile (comme auteur de l'Énéide), Lucain, } \\
\text { Stace... }\end{array}$ \\
\hline
\end{tabular}

Le savoir communiqué n'est pas n’importe quel savoir: il s'agit de “vérités importantes» ou de "quelque art utile à la vie», pour citer une des premières définitions du genre ${ }^{3}$. Si Nicandre écrit un poème sur les poisons et les serpents

3 D. de Mairan, Éloge du cardinal de Polignac, Paris, I747: “L'Anti-Lucrèce est un poème latin du nombre de ceux qu'on appelle didactiques, parce qu'ils ont pour but d'enseigner des vérités importantes ou quelque art utile à la vie. » 
vénéneux, c'est parce que la mort par empoisonnement était la première cause de mortalité dans l'Antiquité; si Virgile écrit les Géorgiques, c'est pour soutenir la politique agricole d'Auguste. Il n'est pas difficile de reconnaître, derrière ce discours savant, une idée des Lumières : les vérités importantes et les arts utiles doivent être communiqués aux hommes. À la poésie de prêter à la communication de ce savoir son charme et sa douceur ${ }^{4}$.

Outre l'absence, dans l'Antiquité, de la notion de "poésie didactique», ce discours moderne sur la poésie didactique antique se heurte à deux problèmes. D’abord, la plupart des œuvres illustrant ce que les Modernes appellent «poésie didactique» sont des poèmes épiques, c'est-à-dire des poèmes en hexamètres. Mais ils ne sont pas considérés comme des épopées, parce que, dans le langage moderne, une épopée est censée être un poème narratif et fictionnel, «racontant les exploits des dieux ou des héros de façon grandiose». Nous avons vu que l'opposition entre "didactique et «épique » recouvrait une opposition entre « enseigner » et «raconter». Or cette dernière opposition n'est pas pertinente pour l'Antiquité. Personne n'y opposait Homère et Hésiode comme nous le faisons. En effet, pour la plupart des Anciens, et jusqu'à la fin de l'Antiquité, Homère est le père de tout savoir et de toute sagesse. Si Hésiode lui est parfois associé dans ce rôle, il n'est jamais question de lui contester la primauté: Homère est l'éducateur de la Grèce, dès l'origine, comme le dit Xénophane 5 . C'est à ce titre que d'autres sages compositeurs de poèmes épiques, comme Parménide ou Empédocle, rivaliseront avec lui. Et c'est à ce titre qu'il sera attaqué par Pythagore ou Héraclite, et bien sûr par Platon.

Aucun, cependant, ne réussira à le détrôner, comme le montre le relief hellénistique du sculpteur Archélaos de Priène, au bas duquel les personnifications de muthos, d'historia, de poiesis, de tragodia, de komodia, mais aussi de phusis, d'arètè, de mnèmè, de pistis et enfin de sophia, viennent sacrifier un taureau à Homère, assis sur un trône (comme Zeus en haut du relief), devant Chronos et Oikoumenè 6 .

Cette idée réapparaîtra en Europe avec les humanistes: pour Melanchthon, “Homère enseigne ce qui est juste et utile, mieux que Chrysippe et Crantor». Après plusieurs exemples, il ajoute qu'il a voulu montrer que la connaissance

4 J. Warton, Reflections, p.292: "To render instruction amiable, to soften the severity of science, and to give virtue and knowledge a captivating and engaging air, is the great privilege of the didactic muse. »

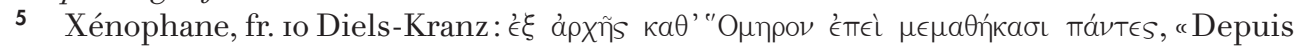
le commencement, tout le monde apprend d'après Homère...»).

6 P. Zanker, Die Maske des Sokrates: Das Bild des Intellektuellen in der antiken Kunst, Munich, I995, p. I54-157. 
des bons auteurs ne forme pas seulement la langue, mais aussi le cœur ${ }^{7}$. Elle est encore défendue au début du XvıI' ${ }^{\mathrm{e}}$ siècle par un philosophe libre penseur, Thomas Collins ${ }^{8}$.

En dehors des poèmes d'Homère, les «mythes» en général sont source d'enseignement et de savoir: directement, par leur valeur «sapientielle» et, dirionsnous aujourd'hui, “scientifique» et «philosophique», par leur exemplarité éthique et esthétique; indirectement, par les explications (scholies, gloses, allégories) auxquels il donnent lieu: à partir de poèmes comme les Argonautiques d'Apollonius de Rhodes ou la Thébaïde de Stace, on pouvait faire le tour encyclopédique de toutes les connaissances. C'est la raison pour laquelle la Bibliothèque d'Apollodore, immense encyclopédie mythographique, s'ouvrait par cette épigramme au lecteur':

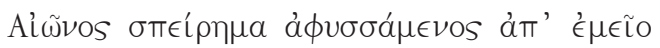

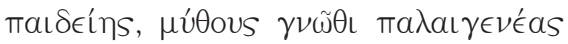

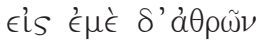

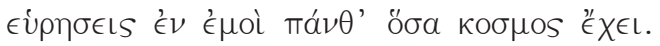

De moi tu tires la semence de l'éternel savoir: la connaissance des légendes antiques.

$[\ldots]$ Regarde-moi, en moi tu trouveras tout ce que contient l'univers.

Pour un Moderne, il peut sembler étonnant qu'un recueil mythographique prétende offrir à son lecteur non pas du plaisir, mais du savoir, et qui plus est le

7 Melanchthon,Declamationes, III, Eloquentiae encomium, p.36-37 (cité par E.R. Curtius, La littérature, p.332): Nec dubitarim adfirmare, quod Horatius censuit, Homerum, quid rectum, quid utile sit, melius Chrysippo et Crantore docere. [...] Neque enim plures Homeri locos his attingere visum est. Tantum hos indicaui, ut studiosis adulescentibus fidem facerem bonorum scriptorum cognitione non os tantum ac linguam, sed pectus etiam formari.

8 R. Bentley, Remarks upon a Late Discourse of Free-Thinking, Cambridge, I713: Homer's Iliad he admires, as the epitome of all arts and sciences. [...] Be it so. [...]. And yet I am apt to fancy, if our author had no better an artist than the old poet for his shoes, he would be as sorry a free-walker as he is now a free-thinker. To prove Homer's universal knowledge a priori, our author says, He designed his poem for eternity, to please and instruct mankind. Admirable again: eternity and mankind: nothing less than all ages and nations were in the poet's foresight. Take my word for it, poor Homer, in those circumstances and early times, had never such aspiring thoughts. He wrote a sequel of songs and rhapsodies, to be sung by himself for small earnings and good cheer, at festivals and other days of merriment. [C'est plus de 500 ans après que Pisistrate fit réunir "the loose songs»]. He no more thought, at that time, that his poems would be immortal, than our free-thinkers now believe their souls will.

9 Photius, Bibliothèque, cod. ı86, I42 $a-b$. 


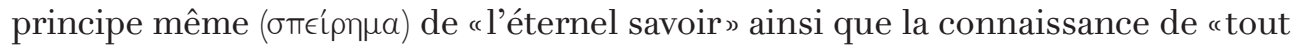
ce que contient l'univers ». Mais c'est que cette culture ignorait les oppositions

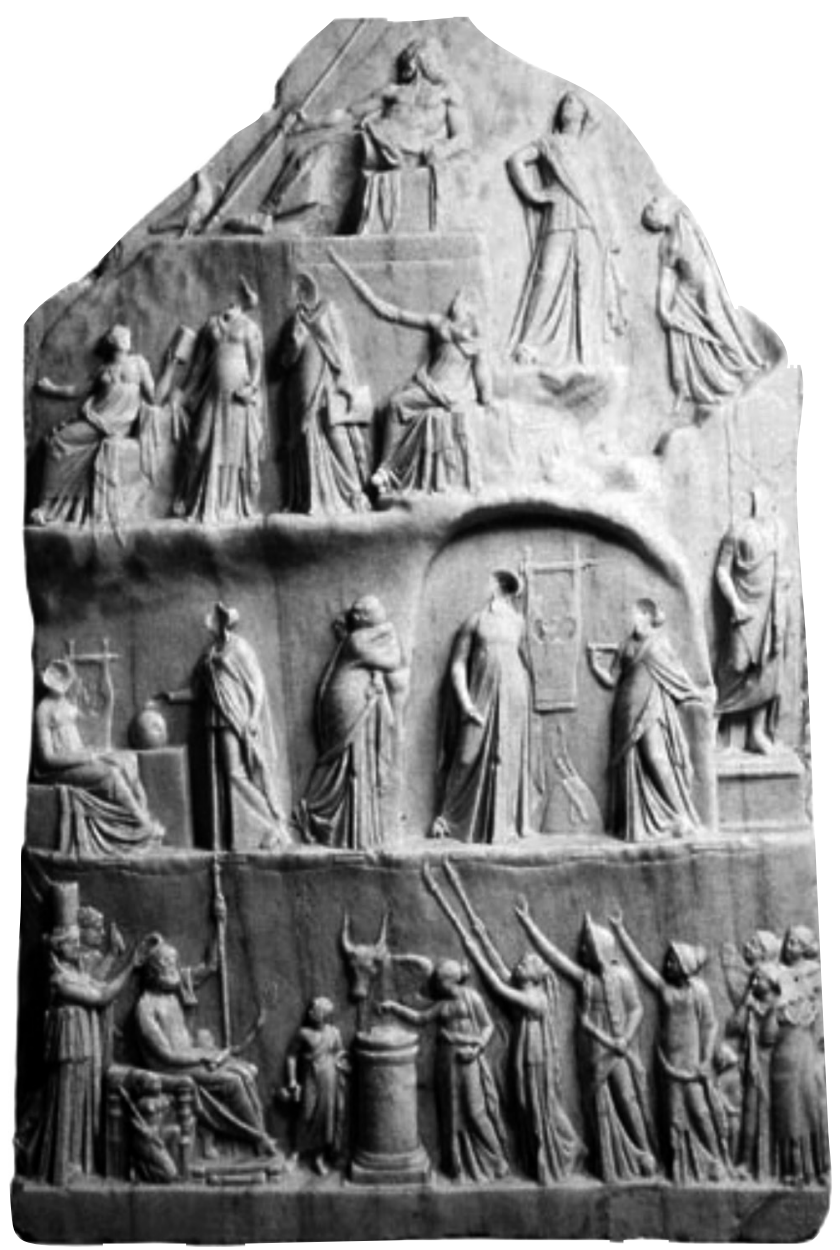

Fig. I: Le bas relief hellénistique d'Archélaos de Priène, British Museum

qui nous sont familières - du « mythe » et de la science, de l'imaginaire et du rationnel, du plaisir et de l'instruction. Les mythes étaient des savoirs: à ce titre, ils enseignaient, exactement comme les savoirs antiques auxquels, contrairement aux mythes, nous reconnaissons encore le titre de savoir: agriculture (nous avons des écoles d'agriculture), astronomie, médecine, philosophie. De ce point de vue, un poème ayant une matière mythique, comme l'Iliade ou les Argonautiques, l'Énéide ou les Métamorphoses, est tout autant «didactique » que Les Travaux et les Jours ou les Phénomènes d'Aratos. 
$\mathrm{Au}$ moins en ce qui concerne la poésie grecque préclassique, il serait difficile de trouver des exemples de poètes qui ne soient pas en même temps des sages, des «maîtres de vérité»: Sappho, Archiloque, Alcman (fragment cosmogonique), Tyrtée, Pindare, Simonide, sans parler des poèmes à chanter dans les banquets de Solon ou de Théognis, qui sont eux aussi destinés indissociablement à charmer qu'à enseigner. C'est une autre opposition paradigmatique moderne, celle des lettres et des sciences, qui empêche d'apercevoir ce fait massif qu'en général, les poètes enseignent. Mais ils enseignent des savoirs que les Modernes ne reconnaissent pas comme savoirs: outre les mythes, ils enseignent les savoirs de l'amour, du banquet et du vin, les savoirs éthiques du guerrier, les chants et les danses des chœurs.

Ainsi, l'opposition entre une poésie didactique et une poésie épique, c'est-àdire entre une poésie qui enseigne et une poésie qui raconte, n'est pas tenable, car elle repose sur une conception du savoir dont les définitions, les classements, les hiérarchies, sont étrangers à l'Antiquité, mais aussi au Moyen Âge.

La deuxième difficulté à laquelle se heurte le discours moderne sur la poésie didactique, c'est que l'association du savoir et de l'utilité, évidente pour des Modernes, ne l'était pas pour les Anciens, et encore moins lorsqu'il s'agissait de poésie. On lit couramment, nous l'avons dit, que les «poèmes didactiques » hellénistiques cherchaient à faciliter la communication de tel ou tel savoir (astronomie, médecine...) en le rendant plus attrayant, grâce à la poésie.

En réalité, les traités en prose suffisaient amplement. On apprenait l'astronomie dans Eudoxe de Cnide. Pourquoi alors le roi Antigone le Borgne a-t-il commandé son poème à Aratos? La réponse se trouve dans la Vie d'Aratos ${ }^{10}$ :

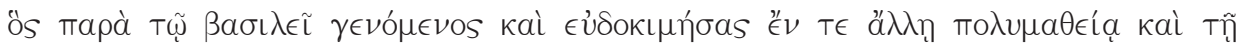

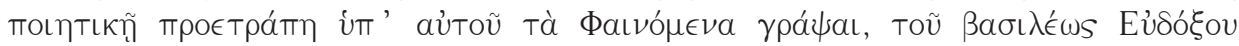

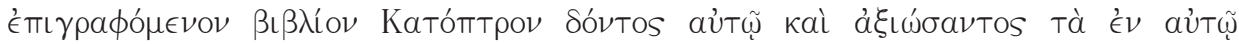

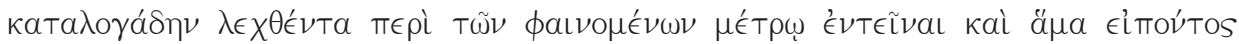

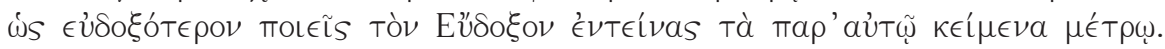

[Aratos], parvenu auprès du roi, se fit connaître par son savoir, mais en particulier par son savoir en matière de poétique. Le roi l'exhorta à écrire les Phénomènes en lui donnant le livre d'Eudoxe. Il jugeait bon que ce qui y était dit en manière de catalogue sur les phénomènes soit mis en vers, ajoutant ceci: «En mettant ses mots en vers, tu rendras Eudoxe plus illustre.»

Il s'agissait donc, non pas de mettre la poésie au service de la prose, la littérature au service de la science, la fiction au service du vrai, mais de prendre une certaine

10 Arati Vita I, 38-43 Martin. 
matière, en l'occurrence “Eudoxe », et de la travailler de façon à en faire une œuvre d'art. Travailler à en faire une œuvre d'art, c'est lui donner de l'enargeia, et lui donner de l'enargeia, c'est rivaliser avec Homère, le maître de l'enargeia "'. C'est pourquoi les Phénomènes se présentent comme une série d'ecphraseis mythologiques des constellations ${ }^{12}$. Comme le dit Manilius, le ciel d'Aratos est une longue fabula. Rien n'empêche qu'on se serve de ce poème pour enseigner l'astronomie, par exemple en corrigeant ses erreurs ${ }^{13}$. Mais Aratos n'a pas composé son poème pour enseigner. Il a voulu faire une œuvre d'art. C'est aussi pourquoi Aratos n'a pas à être lui-même un astronome, pas plus que Nicandre ne devait être agriculteur et médecin pour composer ses Géorgiques et ses Thériaques.

Le fonctionnement des Phénomènes est donc exactement l'inverse de ce qu'en disent les tenants de la catégorie de “poésie didactique : il ne vise pas à enseigner à partir de la poésie ou grâce à la poésie; il vise à faire une œuvre d'art à partir d'un champ du savoir, d'être par son art, en tant que poète, à la hauteur de sa matière. C'est là-dessus que portent tous les jugements sur l'œuvre d'Aratos: il s'agit de

11 Nicandre de Colophon, Thériaques, 957-958.

12 Cf. encore H.-I. Marrou, Histoire de l'éducation dans l'Antiquité, Paris, I948, t. I, p. 275):

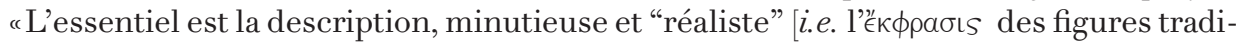
tionnellement prêtées aux constellations : [Aratos] nous montre Persée (248-253), soutenant sur ses épaules son épouse Andromède, tendant la main droite vers la couche de sa belle-mère (Cassiopée), s'élançant d'un pas rapide en soulevant un nuage de poussière (il s'agit en fait d'un amas d'étoiles que présente cette région du ciel) [...] Même anthropomorphisme dans la description des levers et des couchers des constellations (559-732), qui fait suite à une brève évocation des planètes et de la sphère céleste (454558). " Pour une étude plus développée des Phénomènes comme «astronomical ecphrasis», cf. M. Semanoff, “Undermining Authority: Pedagogy in Aratus' Phaenomena », in M.A.Harder, R.F.Retguit, G.C. Wakker (éd.), Beyond the Canon, Leuven, 2006, p.3І3-3г8.

13 De fait, bien que les erreurs contenues dans son poème fussent connues (cf. e.g. Hipparque, in Aratiet EudoxiPhaenomena, I, 2-8 Manitius ; Posidonius, fr. 48 EdelsteinKidd), il était commenté par des mathématiciens et des astronomes comme Attale de Rhodes et Hipparque lui-même, et servait de manuel d'astronomie (cf. le manuel du Stoïcien Géminos de Rhodes). Cf. H.-I. Marrou, Histoire de l'éducation, t. I, p. 275 : « Tel qu'il se présente, le poème d'Aratos n'a rien de mathématique ; pas de chiffres, quelques indications bien sommaires concernant la sphère céleste, son axe, les pôles (v. I9-27) [...] [Le] commentaire [d'Aratos] se bornait à une introduction très sommaire à la sphère [mais] était avant tout littéraire et s'étendait complaisamment sur les étymologies et surtout sur les légendes mythologiques suggérées par la description d’Aratos. »Ce qui comptait n'était pas la “ vérité » (au sens scientifique, moderne, du mot), mais la beauté du savoir. 
savoir s'il réussit ou non à faire une œuvre d'art qui s'élève à la même hauteur que sa matière ${ }^{14}$.

De la même façon, si à Rome Cicéron, Varron d'Atax, Helvius Cinna, Ovide, Germanicus, Aviénus, le père de Stace ${ }^{15}$, l'empereur Gordien ${ }^{16}$, sans oublier le poète anonyme de l'Aratus Latinus et de «nombreux autres », selon saint Jérôme, font chacun à leur tour des «traductions » d'Aratos, ce n'est pas pour «communiquer» le savoir d'Aratos, ce n'est pas dans le but de corriger une traduction précédente pour la rendre plus exacte, mais dans le but de rivaliser avec d'autres poèmes latins dans l'entreprise esthétique consistant à faire une œuvre plus belle que la précédente, et qui soit à Rome une œuvre d'art équivalente aux Phénomènes ${ }^{17}$.

Il en va de même pour Nicandre de Colophon, dont nous avons perdu les Métamorphoses et les Géorgiques, mais dont nous avons encore deux poèmes étonnants : les Thériaques, qui portent sur les serpents venimeux, et les Alexipharmakes, qui portent sur les remèdes aux poisons. Nicandre n'a pas composé ces deux poèmes parce que la mort par poison était la cause de mort la plus fréquente dans l'Antiquité $^{18}$. Comme Aratos avec la matière astronomique, Nicandre a fabriqué une œuvre d'art en prenant pour matière le savoir médical. Ici aussi, faire une œuvre d'art, c'est faire un poème animé par l'enargeia et bourré d'érudition. Ici aussi, c'est devenir un émule d'Homère, comme en témoigne la sphragis des Thériaques:

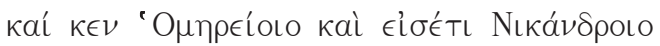

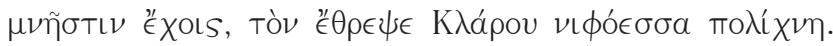

Et de Nicandre l'homérique tu pourras à jamais te souvenir, qui grandit dans la blanche bourgade de Claros.

14 Cic., De or. I, 69; Quintilien X, I, 55: Arati materia motu caret, ut in qua nulla varietas, nullus affectus, nulla persona, nulla cuiusquam sit oratio; sufficit tamen operi cui se parem credidit. "En ce qui concerne Aratos, sa matière manque d'animation: on n'y trouve aucun chatoiement, aucun affect, aucun personnage, aucun discours; et pourtant, il arrive à se hisser à la hauteur de son sujet. »

Silv. 5, 3, I9-23.

16 SHA, Gord., III, 2.

17 M. Pierre, “Rome dans la balance. La poésie augustéenne imite-t-elle la poésie grecque? », in F. Dupont, E. Valette-Cagnac (éd.), Façons de parler grec à Rome, Paris, 2005, p. 229-254.

18 J.-M. Jacques, auteur d'une remarquable édition des deux épopées de Nicandre de Colophon sur les poisons (Thériaques et Alexipharmaques), explique que la composition du poème par le fait que la mort par poison était la plus grande cause de mortalité dans le monde antique (p.3i). 
La pragmatique est donc là encore esthétique: c'est pourquoi, loin de faciliter la communication scientifique, le poème au contraire la suspend. Le texte de Nicandre, conformément à l'esthétique de l'épopée alexandrine, est extrêmement obscur. Dès lors, comme l'ont bien vu les éditeurs anglais de Nicandre, "The victim of snake-bite or poison who turned to Nicander for first-aid would be in sorry plight ${ }^{19}$ ». Les poèmes de Nicandre ne sont donc pas des manuels toxicologiques versifiés, pour être mieux mémorisés ${ }^{20}$. Ils ne sont pas destinés à communiquer (les traités des médecins suffisaient) mais à proposer à l'admiration du public une œuvre d'art exceptionnelle.

Dès lors, comme pour l'opposition Homère-Hésiode, il n'y a pas non plus d'opposition entre Aratos et Nicandre et les autres poètes épiques hellénistiques. Aratos et Nicandre sont désignés comme des poètes épiques, au même titre qu'Apollonius de Rhodes ${ }^{21}$. Il y a une distinction de «matière»: l'un chante les constellations, l'autre les poisons, l'autre l'expédition des Argonautes. Mais ils font la même chose: cette distinction de matière ne correspond pas à une distinction pragmatique. C'est pourquoi un même poète pouvait écrire un poème à matière mythologique et un poème à matière agronomique. C'est pourquoi ils n'ont aucun mal à insérer dans leurs poèmes des séquences venues d'une matière différente, pour montrer qu'ils sont capables de composer des poèmes sur des matières différentes ${ }^{22}$.

Il n'y a rien de plus étonnant pour les lecteurs modernes que le succès remporté dans l'Antiquité par ces poèmes hellénistiques à matière «scientifique ». Comment pouvait-on se passionner pour les Phénomènes d'Aratos ou les Alexipharmakes de Nicandre? Pourquoi les Romains rivalisaient-ils de traductions d'Aratos ou de vers sur les éruptions de l'Etna? Lucilius, le destinataire de Sénèque, ne pourra pas s'abstenir de chanter l'Etna, “ce lieu de tous les poètes » (Lucrèce, Virgile, Ovide...): Quid tibi do ne Aetnam describas in tuo carmine, ne hunc sollemnem omnibus poetis locum adtingas?

19 A. L. F. Gow \& A. F. Shofield, Nicander. The Poems and Poetical Fragments, ig53, p. I8.

20 La plupart des recettes médicales métriques (Galien en cite beaucoup) destinées à la mémorisation ne sont pas en vers épiques, mais souvent en trimètres iambiques, le mètre le plus proche, selon Aristote, du langage parlé (Poet. I449a24). Cf. C. Fabricius, Galens Exzepte aus älteren Pharmakologen, Berlin-New York, I972, p.54. Les recettes en hexamètres semblent souvent des poèmes difficiles à interpréter, donc des jeux savants, où nous avons affaire à une pragmatique ludique et/ou esthétique et non médicale. Cf. e. g. la recette du thériaque d'Andromaque.

21 Dans Quintilien (X, I, 55), Aratos est cité parmi les epici. Chez Athénée (I26 b = fr. 68 des Géorgiques), Nicandre est désigné comme “ faiseur d'épopées » ('̇тотоь́́s) riches en mots

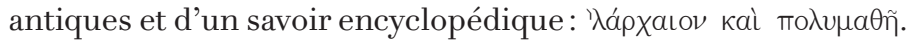

22 Cf. e. g. le long passage sur la crue du Nil dans Lucain. 
C'est notre culture esthétique qui nous empêche d'accepter qu'on ait pu trouver «beau» un poème chantant les serpents venimeux et les remèdes contre les poisons, qu'on puisse prendre plaisir et s'amuser au savoir sans chercher à en faire son profit. Le savoir pour les Modernes n'a pas de valeur esthétique en tant que savoir. Et la poésie didactique, en tant que catégorie moderne, repose justement sur l'idée qu'il faudrait «l'habilller» poétiquement pour le rendre attractif. Dans l'Antiquité classique et hellénistique, le savoir en tant que tel est beau, objet d'émerveillement et de plaisir: c'est pour nous un monde étrange où un volcan fait venir l'eau à la bouche (Aut ego te non novi aut Aetna tibi salivam movet, dit Sénèque à Lucilius), où un roi, pour complaire à ses amis, leur offre des herbes comestibles infectées de sucs vénéneux ${ }^{23}$; où les Indiens peuvent offrir à Auguste un jeune homme sans bras, mais capable de tirer à l'arc avec ses pieds ${ }^{24}$; un monde où l'on est prêt à mourir pour posséder le cheval de Diomède, bien qu'il porte la mort à ses possesseurs l'un après l'autre ${ }^{25}$. Les mirabilia ne sont pas une partie du savoir. Ils sont le savoir.

La valeur du savoir réside pour les Modernes moins dans sa beauté que dans son utilité. C'est pourquoi les commentateurs qui veulent «sauver» les poèmes de Nicandre parlent de son utilité. C'était l'inverse dans l'Antiquité. Les savoirs encyclopédiques de la raı́fía sont des savoirs inutiles, au sens où ils fonctionnent dans un espace voué au loisir et à la détente, au jeu et à la beauté. L'idée qu'il faut privilégier l'utilité pratique des savoirs n'était pas absente dans l'Antiquité, mais minoritaire.

C'est cette pragmatique esthétique que nous retrouvons à Rome avec les poèmes dits «didactiques » de Lucrèce, Virgile ou Manilius. Il s'agit à la fois non pas de communiquer un savoir mais, pour répondre à une commande (car on ne se lance pas tout seul dans la composition d'un poème épique de cette ampleur), de faire une œuvre d'art (illustrare) avec une certaine matière relevant des savoirs lettrés (les écrits d'Épicure, les Travaux et les Jours, l'astronomie, l'astrologie...). Il s'agissait de poètes professionnels: Lucrèce n'était pas plus philosophe que Virgile agronome. D’après une petite épopée, la Ciris, dans laquelle un poète invite le noble Valerius Messalla à lui passer commande d'une grande épopée philosophique, il est possible que les poètes aient offert à de riches Romains de petits poèmes pour montrer leur talent et obtenir une commande importante. C'est ainsi que Lucrèce composa son De rerum natura pour C. Memmius, un sénateur qui

23 Justin 36, 4, 3 s.; Plut., Démétr. 20, 3 (voir Nicandre, dans Budé II, n. 24). Son zèle pour la pharmacologie à base de plantes: Galien I2, 25I, 3 s. K.

24 Dion Cassius, 54, 9.

25 Aulu-Gelle, III, 9 . 
voulait associer à son nom le prestige d'Épicure (auctoritas Epicuri), ou encore que Virgile composa ses Géorgiques pour Mécène. Le prologue du livre III illustre bien la nécessité, pour le poète épique, de trouver une matière à mettre en lumière ${ }^{26}$ :

Cetera quae vacuas tenuissent carmine mentes, omnia iam volgata: quis aut Eurysthea durum aut inlaudati nescit Busiridis aras?

[...] Temptanda via est qua me quoque possim

tollere humo victorque virum volitare per ora.

Toutes les matières capables de capter les esprits ont déjà été enseignées. Qui ignore le terrible Eurysthée ou les autels de Busiris? [...] Je dois donc tenter une route par laquelle moi aussi je puisse me hisser du sol et, victorieux, voler dans les airs.

On voit bien ici que le choix de la matière « géorgique» ne correspond pas à une nécessité intérieure ou à un projet intellectuel, scientifique, politique. Il s'agit de trouver une matière qui permette à l'artiste de montrer ce dont il est capable. Or comme tout a déjà été dit, comme les autres poètes ont déjà chanté la mythologie, il lui faut trouver une matière nouvelle. Il ne s'agit pas de prendre au pied de la lettre ce que dit ici Virgile - il n'était pas le premier à chanter la matière des Géorgiques - mais de repérer et de retrouver ici la pragmatique esthétique de la poésie hellénistique. Le témoignage de Sénèque vient encore le confirmer ${ }^{27}$ :

Vergilius noster [...] non quid verissime sed quid decentissime diceretur aspexit, nec agricolas docere voluit sed legentes delectare.

Notre Virgile ne visait pas la stricte vérité, et il n'a pas voulu instruire les paysans, mais donner du plaisir à ceux qui lisent.

On retrouve ce même dispositif esthétique, où le poème doit se hisser à la hauteur de sa matière, chez Manilius ${ }^{\mathbf{2 8}}$ :

Hunc mihi tu, Caesar, [...]

das animum viresque facis ad tanta canenda. [...].

Bina mihi positis lucent altaria flammis,

ad duo templa precor duplici circumdatus aestu

carminis et rerum. Certa cum lege canentem

mundus et immenso vatem circumstrepit orbe

vixque soluta suis immittit verba figuris.

26 Virgile, Géorgiques III, 3-9.

27 Sénèque, Lettres à Lucilius $86, \mathrm{I} 5$.

28 Manilius, Astronomiques I, 7-24. 
Ce courage, c'est toi qui me le donnes, César, et les forces qu'il faut pour chanter des choses si grandes. [...] Devant moi les flammes éclairent deux autels, j'adresse mes prières à deux temples, entouré par la double effervescence du poème et de l'univers. Autour du prophète qui chante un chant réglé, l'orbe du cosmos immense résonne, qui même à la prose permet à peine de formuler ses figures.

Cette pragmatique esthétique réapparaît à la Renaissance, avec des poèmes chantant les constellations, l'élevage des vers à soie, le jeu d'échecs, ou encore la syphilis. Le poème que lui consacre Fracastor a un immense succès : là encore, nous avons affaire à une culture très différente de la nôtre; à la fin du XvIII ${ }^{\mathrm{e}}$ siècle, le passage le plus aimé de Lucrèce est la description de la peste à Athènes ${ }^{29}$.

De même, on retrouve à la Renaissance le même système de commande que nous avons vu plus haut avec la Ciris, avec le passage du petit poème au grand poème: Léon X, ayant approuvé le poème de Vida sur les vers à soie, commande la Christiade.

Personne ne parle encore de poésie didactique: ni Du Bellay ni Ronsard ni Le Tasse ni, plus tard, Boileau. Il y a des épopées, qui portent sur la nature ou sur les hauts faits des héros, mais cette distinction de matière, comme dans l'Antiquité, n'est pas une distinction générique, pragmatique: ce sont, avec des matières différentes, les mêmes types d'objets.

Il faut cependant distinguer à la Renaissance entre les poèmes qui renouent avec la tradition esthétique des poèmes antiques et ceux qui prétendent agir sur la conscience des lecteurs, et qui se rattachent à la tradition médiévale du poème sacré. C'est là aussi un signe de ce que la catégorie de “poésie didactique» est ancrée dans la culture intellectuelle moderne: jamais ne sont donnés comme exemples tous les poèmes médiévaux qui pourtant voulaient enseigner (Alain de Lille, Bernard Silvestre, Dante, etc.), parce qu'il s'agit d'un savoir religieux, et donc pas d'un savoir véritablement considéré comme tel par les Modernes.

Il faut attendre l'époque des Lumières pour que naisse véritablement l'idée que la poésie peut communiquer le savoir plus facilement et plus agréablement. Il n’est alors plus question de pragmatique esthétique. Si le cardinal de Polignac fait un antiLucrèce, c'est parce que, dit-il, en discutant avec Pierre Bayle, il s'est rendu compte du danger que l'épicurisme faisait courir aux hommes de son temps ${ }^{30}$. On n’imagine pas aujourd'hui le succès de poèmes comme l'Essayon man de Pope, les discours en vers de Voltaire («discours » en vers justement, et non poèmes), ou encore les poèmes

29 J. Warton, Reflections, p.420: "The plague with which the whole poem concludes being more known and perhaps more read than any other part of it, I shall not point out any other particular passages. »

30 MaIran, Éloge. 
de l'abbé Delille ${ }^{31}$. Mais la tradition de la “poésie didactique », c'est-à-dire, pourraiton dire en paraphrasant Péguy, de la poésie «de propositions » (philosophiques ou théologiques ${ }^{32}$ reçut probablement un coup fatal avec le romantisme et l'idéalisme allemand, autonomisant la poésie comme forme de savoir à part entière, et non pas comme véhicule ou moyen d'un savoir aussi prosaïque que l'art des jardins, le fonctionnement du baromètre, les vertus du cidre ou de l'eau, la circulation du sang, “l'art de préserver sa santé». Hegel, qui déteste la poésie didactique, cite souvent Schiller ou Goethe. Il est significatif que Flaubert, dans ses notes sur l'Esthétique, relève l'arbitraire qu'il y a à classer ensemble Lucrèce, Virgile et l'abbé Delille: «Il aurait fallu montrer pourquoi les deux premiers sont si au-dessus du troisième ${ }^{33}$ ! Désormais, la poésie n'était plus la servante de la science: elle redevenait, en un sens, une part du savoir.

\author{
Pierre Vesperini \\ Université Paris 8 \\ 2, rue de la Liberté \\ 93526 Saint-Denis \\ pvesperini@gmail.com
}

31 Le chant VI de Delille sur les trois règnes est commenté par Cuvier.

32 C. PÉGuy, “L'Ève de Péguy », in Euvres complètes, Bibliothèque de la Pléiade, III, Paris, I94I, p. I235: “Il a été assez heureux pour transporter dans ses vers cette intègre probité qui paraissait ne pas pouvoir quitter la prose. [...] L'auteur [...] a fait des vers de poètes avec une sorte de marbre de prose. Aussi avons-nous dans ce poème jusqu'à des propositions de philosophie et de théologie réduites en des vers d'une telle justesse technique qu'il faudrait peut-être remonter jusqu'au De natura rerum pour en trouver d'une telle sévérité. » Et en note: “Il ne faudrait pas, si l'on veut être juste, oublier les très beaux vers philosophiques de Sully-Prudhomme, notamment dans La Justice, qui sont eux aussi des vers de propositions ».

33 Flaubert, «Notes sur l'Esthétique de Hegel». Voici le texte complet: «Hegel pense que la poésie didactique ne doit pas être comptée parmi les formes propres à l'art. Enfin le fond et la forme sont ici complètement isolés. - La forme artistique ne peut être rattachée au fond que par un rapport tout extérieur parce que l'idée, l'enseignement, s'adresse avant tout à la raison et à la réflexion et que dès lors sa condition essentiellement prosaïque ne peut être poétiquement développée mais simplement revêtue d'une forme poétique, malgré l'habileté de détails. Lucrèce, Virgile - Delille! Il aurait fallu montrer pourquoi les deux premiers sont si au-dessus du 3 e ! Ces notes de Flaubert ont été publiées dans Dix ans de critique. Notes inédites de Flaubert sur L'Esthétique de Hegel, textes réunis et présentés par Gisèle Séginger, Paris, Lettres Modernes Minard, “Gustave Flaubert, 5 », 2005, p. 247-33o. 